\title{
Finite Sum Evaluation of the Gauss Hypergeometric Function in an Important Special Case
}

\author{
By John Detrich and Robert W. Conn
}

\begin{abstract}
We present explicit results expressing the Gauss hypergeometric function $F(l+1, m+1 / 2 ; n+3 / 2 ; z)$ as a finite sum in the general case where $l, m$, and $n$ are integers.
\end{abstract}

In a recent paper dealing with an integral which arises in the evaluation of first Born scattering matrix elements [1] we obtained a result which suggests that a Gauss hypergeometric function of the form $F(l+1, m+1 / 2 ; n+3 / 2 ; z)$ can always be expressed in finite terms if $l, m$ and $n$ are integers. Functions of this type occur often enough in mathematical physics to be of general interest, but to our knowledge no discussion of the general case has been presented. This is the task we take up here.

It is well known that when $l<0$, the hypergeometric series $F(l+1, m+1 / 2$; $n+3 / 2 ; z)$ truncates. The case $n-m<0$ may be disposed of in similar fashion upon application of Euler's identity [2]

$$
F(a, b ; c ; z)=(1-z)^{c-a-b} F(c-a, c-b ; c ; z) .
$$

For the remaining case, we will show that $F(l+1, m+1 / 2 ; n+3 / 2 ; z)$ can be written as

$$
\begin{aligned}
F(l+1, m+1 / 2 ; n+3 / 2 ; z)=A_{l, m, n}(z) \log \left(\frac{1+z^{1 / 2}}{1-z^{1 / 2}}\right)+ & B_{l, m, n}(z), \\
& l \geqslant 0, n-m \geqslant 0,
\end{aligned}
$$

where $A_{l, m, n}(z)$ and $B_{l, m, n}(z)$ are finite expressions in terms of powers of $z$ and $(1-z)$. In case $l=m, F(m+1, m+1 / 2 ; n+3 / 2 ; z)$ can be expressed in terms of the associated Legendre function of the second kind, $Q_{n}^{2 m-n}(y)$, where $y=z^{-1 / 2}$. Hence, Eq. (2) is the generalization of an expression for $Q_{n}^{0}(y)$ established some time ago [3].

As we will show, the function $A_{l, m, n}(z)$ in Eq. (2) is given by

$$
\begin{aligned}
A_{l, m, n}(z)= & \frac{(-1)^{n-m-l} \Gamma(n+1 / 2) \Gamma(n+3 / 2) z^{-n-1 / 2}}{\Gamma(m+1 / 2) \Gamma(n-l+1 / 2) \Gamma(l+1) \Gamma(n-m+1)} \\
& \times F(l-n+1 / 2, m-n ; 1 / 2-n ; z),
\end{aligned}
$$

where $\Gamma(n+1 / 2)$ is the usual gamma function. For $B_{l, m, n}(z)$ it is convenient to

Received June 13, 1977; revised April 3, 1978, June 30, 1978.

AMS (MOS) subject classifications (1970). Primary 33A30. 
specialize to the case

$$
n-m-l \geqslant 0 .
$$

Using Eq. (1), the remaining case may be handled by means of the relation

$$
B_{l, m, n}(z)=(1-z)^{n-m-l} B_{n-m, n-l, n}(z) .
$$

When Eq. (4), applies, we will show that

(6) $B_{l, m, n}(z)=\sum_{p=0}^{-m-1} f_{l, m, n, p} z^{p}+\sum_{p=-m}^{-l-1} g_{l, m, n, p^{2}} z^{p} \sum_{p=-n}^{-m-1} h_{l, m, n, p} z^{p}$,

where it is to be understood that the first sum yields zero if $-m-1<0$, that the second sum yields zero if $-l-1<-m$, and that the third sum yields zero if $-m-$ $1<-n$. The coefficients used in Eq. (6) are given by

$g_{l, m, n, p}=\frac{(-1)^{l+1} \Gamma(n+3 / 2) \Gamma(m-l-1 / 2)(l+1)_{-l-p-1}(l-n+1 / 2)_{-l-p-1}}{\Gamma(m+1 / 2) \Gamma(n-l+1 / 2)(l-m+3 / 2)_{-l-p-1}(-l-p-1) !}$,

$$
h_{l, m, n, p}=\frac{(-1)^{n-m-l} \Gamma(n+1 / 2) \Gamma(n+3 / 2)}{\Gamma(m+1 / 2) \Gamma(n-l+1 / 2) \Gamma(l+1) \Gamma(n-m+1)}
$$

(9)

$$
\times \sum_{q=0}^{p+n} \frac{(l-n+1 / 2)_{q}(m-n)_{q}}{(1 / 2-n)_{q} q !(1 / 2+p+n-q)},
$$

where we use the customary notation

$$
(a)_{p}=a(a+1)(a+2) \cdots(a+p-1)=\Gamma(a+p) / \Gamma(a) .
$$

To establish the results put forward in Eqs. (2)-(9) it is sufficient to prove the results for the case where Eq. (4) applies. Then, by using Euler's identity in Eq. (1), we can immediately dispose of the remaining case. We use Eq. (3) and the power series expansion implied by the relation

$$
\log \left(\frac{1+z^{1 / 2}}{1-z^{1 / 2}}\right)=2 z^{1 / 2} F(1,1 / 2 ; 3 / 2 ; z)
$$

to obtain, after a little rearrangement,

$$
A_{l, m, n}(z) \log \left(\frac{1+z^{1 / 2}}{1-z^{1 / 2}}\right)=\sum_{p=-n}^{-m-1} h_{l, m, n, p} z^{p}+\sum_{p=-m}^{\infty} \alpha_{l, m, n, p} z^{p}
$$

where

$$
\alpha_{l, m, n, p}=C_{l, m, n} \sum_{q=0}^{n-m} \frac{(m+1 / 2)_{q}(m-n)_{q}}{(m-l+1 / 2)_{q} q !(1 / 2+p+m+q)} .
$$


Here we adopt the abbreviation

$$
C_{l, m, n}=(-1)^{l} \Gamma(n+3 / 2)[\Gamma(l+1) \Gamma(n-m+1) \Gamma(m-l+1 / 2)]^{-1} .
$$

Now the result will be established if we can show that

$$
\alpha_{l, m, n, p}=\left\{\begin{array}{ll}
-g_{l, m, n, p}, & p \leqslant-l-1, \\
0, & -l \leqslant p \leqslant-1, \\
f_{l, m, n, p}, & p \geqslant 0
\end{array}\right\}
$$

To demonstrate this, we note that since $p+m \geqslant 0$ is always true, Eq. (13) can be rewritten to give

$$
\alpha_{l, m, n, p}=C_{l, m, n} \int_{0}^{1} d t t^{p+m-1 / 2} F(m+1 / 2, m-n ; m-l+1 / 2 ; t) .
$$

We substitute according to the differential relation [4]

$$
\begin{aligned}
& \frac{d^{l}}{d t^{l}}\left[t^{m-1 / 2} F(m+1 / 2, m-n ; m+1 / 2 ; t)\right] \\
& \quad=(m-l+1 / 2) t^{m-l-1 / 2} F(m+1 / 2, m-n ; m-l+1 / 2 ; t),
\end{aligned}
$$

and note

$$
F(m+1 / 2, m-n ; m+1 / 2 ; t)=(1-t)^{n-m} .
$$

Since Eq. (4) applies, we find

$$
\left\{\frac{d^{q}}{d t^{q}}\left[t^{m-1 / 2}(1-t)^{n-m}\right]\right\}_{t=1}=0, \quad q \leqslant l-1,
$$

so that a repeated integration by parts brings Eq. (16) to the form

With the help of the result

$$
\begin{aligned}
\alpha_{l, m, n, p}= & (-1)^{l} C_{l, m, n}\left[(m-l+1 / 2)_{l}\right]^{-1} \\
& \times \int_{0}^{1} d t t^{m-1 / 2}(1-t)^{n-m} \frac{d^{l}}{d t^{l}} t^{l+p}
\end{aligned}
$$

(21) $\int_{0}^{1} d t t^{p+m-1 / 2}(1-t)^{n-m}=\Gamma(p+m+1 / 2) \Gamma(n-m+1) / \Gamma(p+n+3 / 2)$,

Eq. (20) is easily reduced to yield Eq. (15).

From the standpoint of computation, the 15 relations of Gauss for contiguous hypergeometric functions [5] can be valuable. Hence, it is worth noting that the functions $A_{l, m, n}(z)$ given by Eq. (3) satisfy the same 15 contiguous relations as do the corresponding functions $F(l+1, m+1 / 2 ; n+3 / 2 ; z)$. This can be established in a straightforward manner, utilizing the observation that only 4 of the 15 relations are actually independent. It then follows from Eq. (2) that the functions $B_{l, m, n}(z)$ must also satisfy the same recurrence relations. 
Also worth noting is the relation

$$
\begin{aligned}
& F(\nu+1 / 2, \nu+\mu+1 ; \nu+\mu+\lambda+3 / 2 ; z) \\
&=\frac{(-1)^{\mu} \pi^{1 / 2} \Gamma(\nu+\mu+\lambda+3 / 2)}{\Gamma(\nu+1 / 2) \Gamma(\lambda+1 / 2) \Gamma(\nu+\mu+1) \Gamma(\mu+\lambda+1)} \\
& \times \frac{d^{\nu+\mu}}{d z^{\nu+\mu}}\left\{(1-z)^{\mu+\lambda} \frac{d^{\lambda}}{d z^{\lambda}}\left[z^{-1 / 2} \log \left(\frac{1+z^{1 / 2}}{1-z^{1 / 2}}\right)\right]\right\} .
\end{aligned}
$$

This may be considered in analogy to the result [6]

$$
\begin{aligned}
F(\nu+1, \nu+\mu+1 ; \nu+\mu+\lambda+2 ; z) & \\
& =\frac{(-1)^{\mu+1}(\nu+\mu+\lambda+1) !}{\lambda ! \nu !(\nu+\mu) !(\mu+\lambda) !} \frac{d^{\nu+\mu}}{d z^{\nu+\mu}}\left\{(1-z)^{\mu+\lambda} \frac{d^{\lambda}}{d z^{\lambda}}\left[z^{-1} \log (1-z)\right]\right\} .
\end{aligned}
$$

In the latter result, $\lambda, \mu$, and $\nu$ take nonnegative integer values, and since Eq. (22) is derived in similar fashion, the same restrictions evidently apply there as well.

Acknowledgements. We would like to thank Professor Richard A. Askey for his helpful comments. We also thank the referee for suggesting the inclusion of Eq. (22). This research was partially supported by the U. S. Energy Research and Development Administration.

Nuclear Engineering Department

University of Wisconsin

Madison, Wisconsin 53706

1. J. DETRICH \& R. W. CONN, J. Mathematical Phys., v. 18, 1977, p. 2348.

2. A. ERDELYI ET AL., Higher Trancendental Functions, Vol. I, McGraw-Hill, New York, 1953, p. 64.

3. Ibid., p. 153.

4. Ibid., p. 102.

5. Ibid., p. 57 and p. 103.

6. Ibid., p. 69. 